\title{
Controlling chimera states via minimal coupling modification
}

Cite as: Chaos 29, 051103 (2019); https://doi.org/10.1063/1.5097570

Submitted: 26 March 2019. Accepted: 17 April 2019. Published Online: 07 May 2019

Giulia Ruzzene, Iryna Omelchenko, Eckehard Schöll (D), Anna Zakharova (D), and Ralph C. Andrzejak
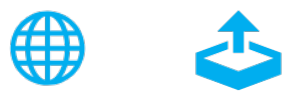

Export Citation

\section{ARTICLES YOU MAY BE INTERESTED IN}

Complexity reduction ansatz for systems of interacting orientable agents: Beyond the Kuramoto model

Chaos: An Interdisciplinary Journal of Nonlinear Science 29, 053107 (2019); https:// doi.org/10.1063/1.5093038

Collective dynamics of globally delay-coupled complex Ginzburg-Landau oscillators Chaos: An Interdisciplinary Journal of Nonlinear Science 29, 053104 (2019); https:// doi.org/10.1063/1.5087188

Synchronization of spiral wave patterns in two-layer 2D lattices of nonlocally coupled discrete oscillators

Chaos: An Interdisciplinary Journal of Nonlinear Science 29, 053105 (2019); https:// doi.org/10.1063/1.5092352

\section{AIP Author Services} English Language Editing 


\title{
Controlling chimera states via minimal coupling modification
}

\author{
Cite as: Chaos 29, 051103 (2019); doi: 10.1063/1.5097570 \\ Submitted: 26 March 2019 . Accepted: 17 April 2019. \\ Published Online: 7 May 2019

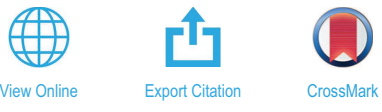

Giulia Ruzzene, ${ }^{1, a)}$ Iryna Omelchenko, ${ }^{2}$ Eckehard Schöll, ${ }^{2}$ (D) Anna Zakharova, ${ }^{2}$ (D) and Ralph G. Andrzejak ${ }^{1,3}$

\begin{abstract}
AFFILIATIONS
'Department of Information and Communication Technologies, Universitat Pompeu Fabra, Carrer Roc Boronat 138 , 08018 Barcelona, Catalonia, Spain

${ }^{2}$ Institut für Theoretische Physik, Technische Universität Berlin, Hardenbergstrasse 36, 10623 Berlin, Germany

${ }^{3}$ Institute for Bioengineering of Catalonia (IBEC), The Barcelona Institute of Science and Technology, Baldiri Reixac 10-12, 08028 Barcelona, Spain
\end{abstract}

Electronic mail: giulia.ruzzene@upf.edu

\begin{abstract}
We propose a method to control chimera states in a ring-shaped network of nonlocally coupled phase oscillators. This method acts exclusively on the network's connectivity. Using the idea of a pacemaker oscillator, we investigate which is the minimal action needed to control chimeras. We implement the pacemaker choosing one oscillator and making its links unidirectional. Our results show that a pacemaker induces chimeras for parameters and initial conditions for which they do not form spontaneously. Furthermore, the pacemaker attracts the incoherent part of the chimera state, thus controlling its position. Beyond that, we find that these control effects can be achieved with modifications of the network's connectivity that are less invasive than a pacemaker, namely, the minimal action of just modifying the strength of one connection allows one to control chimeras.
\end{abstract}

Published under license by AIP Publishing. https://doi.org/10.1063/1.5097570

In networks of oscillators, chimera states are phenomena defined as the coexistence of coherence and incoherence. ${ }^{1,2}$ They were first observed in 2002 by Kuramoto and Battogtokh who studied the dynamics of a ring-shaped network of nonlocally coupled oscillators. ${ }^{1}$ A chimera state is formed when the oscillators spontaneously split into two complementary groups, one displaying an almost synchronous behavior and the other in which the oscillators perform an erratic motion. Thus, the spatial symmetry of the network's equations is broken by the spatiotemporal evolution of its dynamics. Since the initial discovery in ring networks of nonlocally coupled oscillators, chimeras have been observed for a variety of network node dynamics and network coupling topologies. ${ }^{3-9}$ The interest in the study of this fascinating phenomenon grew, thanks to the observation of chimeras in experiments, ${ }^{5,10-16}$ and numerous conceptual links established between chimera states on the one hand and natural and manmade phenomena on the other. ${ }^{17-21}$ Many advances were made in the understanding chimeras from a mathematical perspective (see Ref. 22 and the references therein). For finite-size networks of nonlocally coupled phase oscillators, chimera states are not stable but can collapse to the synchronous state at any moment in time. ${ }^{19,23,24}$ Another finite-size effect on chimera states is the drifting of the groups within the network, which can be characterized as a Brownian motion. ${ }^{25}$ It has been shown that it is possible to control these instabilities of chimera states. ${ }^{19,24,26-30}$ With the expression controlling chimeras, we here mean the interactions with the network aimed to influence the formation, the position, and the collapse of the chimera state. Control methods include closed feedback loops ${ }^{19,24,26,27}$ and open-loops. ${ }^{28-30}$

An open problem is to find the minimal action needed to control chimera states. To address this problem, we choose to act exclusively on the connectivity structure of the network. We develop an open-loop mechanism based on the idea of a pacemaker oscillator. We implement this mechanism selecting one oscillator and gradually eliminating its incoming connections, while maintaining its outgoing connections. We first show that this modification can induce chimeras in cases in which they do not form spontaneously. We then illustrate that the pacemaker attracts the incoherent part of the chimera. Furthermore, removing even a small fraction of the connections of one oscillator or just lowering the strength of one connection is sufficient to achieve control. These results also point to the fact that 
symmetry breaking is an essential part of chimera control. To further strengthen this claim, we show that the opposite of the full pacemaker, i.e., an oscillator with no outgoing links, produces control effects qualitatively similar to what we obtain with the pacemaker. The advantages of our method are its simple implementation and the possibility of controlling chimeras with minimal actions on the network coupling topology. We expect that these aspects will make our method attractive for possible applications in which there is limited access to the system showing chimera states.

\section{INTRODUCTION}

Methods to achieve control of chimera states have been implemented in models featuring different types of oscillators. In 2014, Sieber and co-workers published a closed-loop method for ringshaped networks of phase oscillators, which used a time-dependent phase-lag parameter to prevent chimera states from collapsing to the synchronized state. ${ }^{24}$ Another closed-loop method based on a gradient dynamics that allows one to maintain the position of the chimera state was proposed by Bick and Martens in 2015. ${ }^{26}$ In 2016, Omelchenko et al ${ }^{27}$ developed a feedback control mechanism, called tweezers, to control chimera states in small networks of FitzHughNagumo and Van der Pol oscillators. This method uses two components: a symmetric one to prevent the collapse of chimeras and an asymmetric one to control their position. ${ }^{27}$ The tweezers mechanism was optimized in Ref. 31 allowing to control the size of the domains forming the chimera state and the frequency difference among the oscillators in each domain. Gambuzza and Frasca ${ }^{28}$ used spatial pinning to control the position of chimera states in networks of FitzHugh-Nagumo and phase oscillators. Isele et al. conducted a study about control of the position of chimeras in networks of oscillatory FitzHugh-Nagumo units. ${ }^{29}$ They introduced a barrier of excitable units in the network, which attracts the incoherent region. ${ }^{29}$ In the work by Andrzejak et al., ${ }^{19}$ closed-loop feedback control schemes were used to suppress or promote the collapse of the chimera to the synchronous state in networks of phase oscillators. Recently, the possibility of controlling some features of chimera states in networks of Stuart-Landau oscillators acting on the initial conditions and coupling scheme has been developed by Kalle et al..$^{30}$ It was also proven that it is possible to control not only classical phase chimeras, but also amplitude chimeras which are observed in networks of Stuart-Landau oscillators. ${ }^{7}$ Furthermore, in phase oscillator networks with coupling functions involving higher order harmonics, chimera states can be stabilized without external influence. ${ }^{32,33}$ All these previous studies on control of chimeras rely on modifications of parameters of the oscillators, and in some cases, these changes are made according to information extracted from the system. In real-world applications, however, it might be difficult to alter the individual oscillators that form a network. Closed-loop feedback methods could also result unreliably, for example, when measurements of system features are affected by noise.

With the goal of finding the minimal action needed to control chimeras, we propose here an open-loop control mechanism that avoids these issues and acts uniquely on the coupling topology of the network, leaving unaltered the oscillators' parameters. We consider the Kuramoto-Sakaguchi model of nonlocally coupled phase oscillators in a ring topology. Our control mechanism is based on the idea of modifying the coupling topology so that a pacemaker oscillator is present in the network. A pacemaker is an oscillator that influences the other oscillators to which it is connected but is not influenced by them. In other words, it is an oscillator whose links are all unidirectional in the connectivity structure. Starting from this extreme, we reduce the number of modified links, thus considering modifications of the connectivity that are less and less invasive. We finally push this mechanism to the limit and act only on one link. We first remove it from the connectivity and then gradually increase its strength until we restore the original connectivity. This allows us to find the minimal intervention needed to control chimeras.

In what follows, we first review the model of a ring-shaped network of phase oscillators, then we introduce our procedure to modify the coupling structure of the network with different intensities of the control mechanism. We present results regarding the formation of chimera states and the control of their position. Finally, we conclude with a brief discussion of the results.

\section{COUPLED OSCILLATOR MODEL}

We use a ring-shaped network of $N$ nonlocally coupled phase oscillators [see Figs. 1(a) and 1(c)]. This network is described by the following system of differential equations for the time-dependent

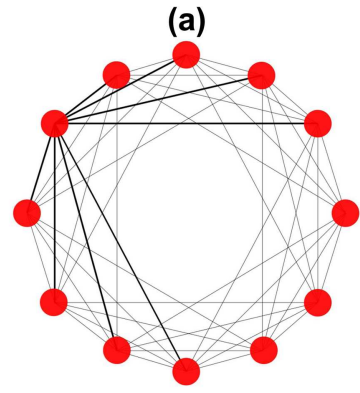

(c)

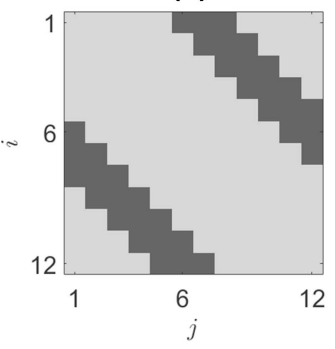

(b)

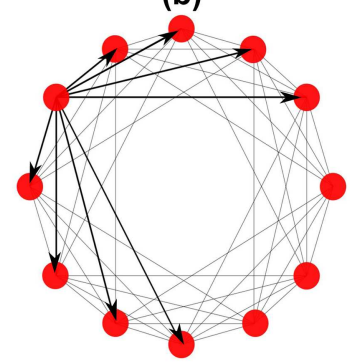

(d)

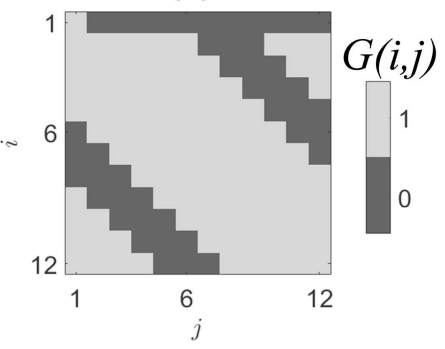

FIG. 1. Implementation of the full pacemaker. In panel (a), we show a network of 12 oscillators that are nonlocally coupled with $b=4$. The links of one oscillator are highlighted to better show the nonlocal coupling configuration. In panel (b), we show how we change the connectivity to implement the pacemaker: we choose one oscillator [the one with highlighted links in panel (a)] and we make its link unidirectional. The corresponding coupling matrices $G(i, j)$ are shown in panels (c) and (d), respectively. 
phases $\phi_{j}(t)$ of the oscillators: $:^{23,25}$

$$
\begin{aligned}
\dot{\phi}_{j}(t) & =\omega-\frac{1}{2 b} \sum_{k=1}^{N} G(j, k) \sin \left(\phi_{j}(t)-\phi_{k}(t)+\alpha\right), \\
G(j, k) & = \begin{cases}1 \text { if } & |j-k| \leq b, \\
0 & \text { otherwise, }\end{cases}
\end{aligned}
$$

where $i, j=1, \ldots, N$. The oscillators' natural frequency $\omega$ is set to be zero without loss of generality. The connectivity matrix $G$ corresponds to a rectangular coupling kernel with broadness $2 b+1 .^{23,25}$ The phase-lag parameter is set to $\alpha=1.46$. $^{23}$ Reflecting the periodic boundary conditions of the network's ring shape, all sums and differences of indexes are to be understood modulo $N$.

To solve the differential equations, we used the 4-th order Runge-Kutta method, with fixed sampling time of $d t=0.05$. We integrated Eq. (1) starting from initial conditions uniformly distributed in the interval $[0,2 \pi)$. To detect chimera states, we adapted an algorithm proposed by Isele et al., ${ }^{29}$ which is based on the global Kuramoto order parameter and the mean phase velocity profiles that characterize chimera states (see Appendix A). Following the terminology introduced in Ref. 19, we refer to the two complementary groups forming the chimera states as the high coherence group (HCG) and the low coherence group (LCG). In Figs. 2(a)-2(c), we show three independent realizations of Eq. (1). Panels (d)-(f) are their corresponding representations in terms of the HCG and LCG.
Panel (a) shows a typical chimera state and illustrates the drift of the LCG and HCG. Panel (b) shows another chimera state that collapses after a short time. Finally, for the realization in panel (c), no chimera is formed and the oscillators synchronize after a short transient.

\section{MODIFYING NETWORK CONNECTIVITY TO CONTROL CHIMERAS}

Our control mechanism acts on the connectivity matrix $G$ defined in Eq. (2). We implement the idea of a pacemaker oscillator in the model in the following way. We decide to have the pacemaker in position $i$, which corresponds to setting to zero all the elements of the $i$-th row of $G$, except for the diagonal entry $G(i, i)$. Accordingly, the $i$-th oscillator does not receive any input and as a consequence it oscillates at a constant angular frequency $\dot{\phi}_{i}(t)=-\sin (\alpha)$. However, since the $i$-th column of $G$ is maintained, this constant frequency is received by all oscillators within the coupling range $b$ of oscillator $i$ [see Fig. 1, panels (b) and (d)]. Subsequently, we implement gradually less invasive modifications of the coupling matrix $G$. Like we just described, in the pacemaker configuration, only the diagonal element is maintained at $G(i, i)=1$. Starting from this most invasive control, we then restore the pair of first off-diagonal elements $G(i, i-1)=G(i, i+1)=1$, then the pair of second off-diagonal elements $G(i, i-2)=G(i, i+2)=1$, etc. This process is continued until we set the elements $G(i, i-b+1)=G(i, i+b-1)=1$. Therefore, at this stage only the elements $G(i, i-b), G(i, i+$ $b)$ remain modified to zero. We refer to the case in which (a)

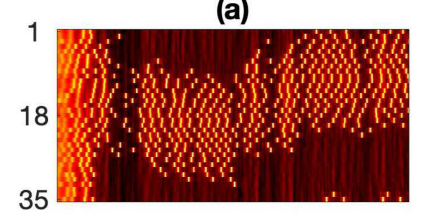

(b)

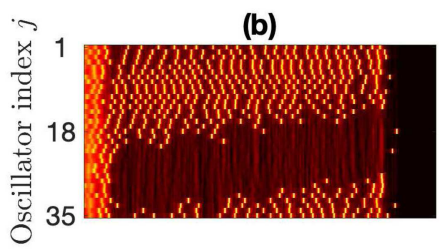

(c)

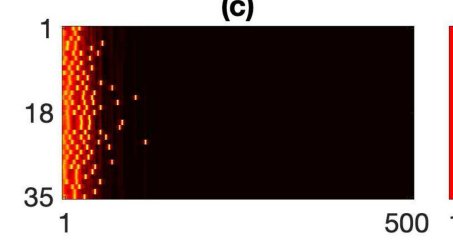

(d)

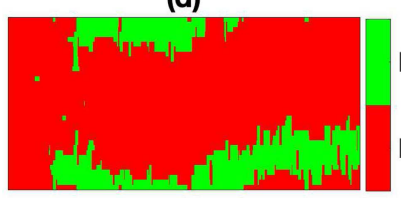

(e)

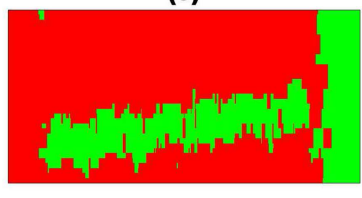

(f) (g)

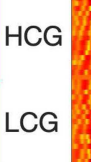

(j)

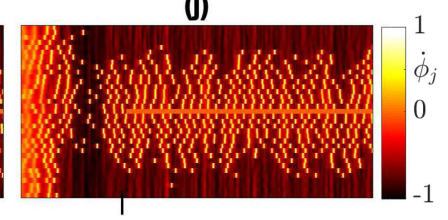

(h)
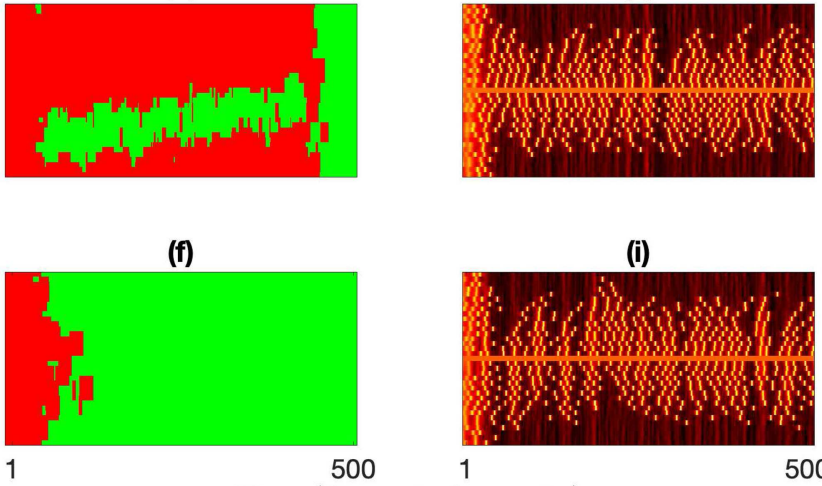

(i)

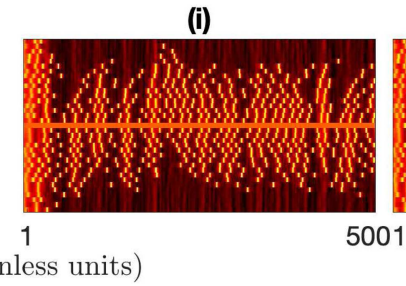

1

Time (dimensionless units)

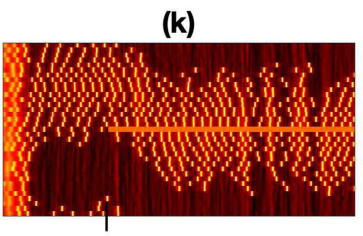

(k)

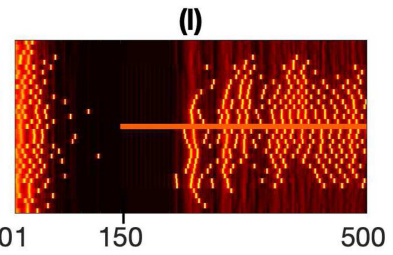

FIG. 2. Uncontrolled chimera states drift along the network over time and may collapse to the synchronous state. The pacemaker stabilizes chimera states. In panels (a)-(c), we display instantaneous phase velocities for three different realizations of Eq. (1) for $N=35, b=12, \alpha=1.46, \omega=0$. Panels (d)-(f) illustrate the division of the corresponding solutions into the high coherence group (HCG) and the low coherence group (LCG). In panels (g)-(i), we display the effects of the presence of a pacemaker in position 18 on the solutions shown in panels (a)-(c), respectively. The pacemaker was activated at the beginning of the simulations. Panels (j)-(I) are analogous to panels (g)-(i), but here the pacemaker was activated after 150 dimensionless time units. 
all coefficients of the $i$-th row of $G$ are set to zero as "full pacemaker" [Figs. 1(b) and 1(d)], and to the intermediate modifications of $G$ described above as "partial pacemaker". The "pacemaker intensity" $\psi$ is defined as the ratio between the number of removed links and the initial number of bidirectional connections of the pacemaker. The lowest possible nonzero value of $\psi$ is $1 / b$, which corresponds to just two unidirectional links of oscillator $i$. Finally, we set $G(i, i-b)=1$ and $G(i, i+b)=\xi$, where $\xi$ is varied from 0 to 1 . That means, for $\xi=1$, the unchanged connectivity matrix $G$ is restored [see Eq. (2)].

The two rightmost columns of Fig. 2 show the effects of the full pacemaker: attracting the LCG and preventing the collapse to the synchronized state. In panels (g)-(i), we start the system with the same initial conditions as in panels (a)-(c), respectively, but now a pacemaker is present in position $i=18$. We see how the pacemaker attracts the LCG. In panel (i), the collapse to the synchronous state is avoided. Panels (j)-(l) show effects analogous to the ones in panels (g)-(i) but now the pacemaker is activated only after 150 time units.

\section{TRIGGERING CHIMERA STATES}

First, we use a pacemaker to induce chimera states for parameters and initial conditions for which they do not form spontaneously [see again Fig. 2, panels (c), (f), (i), and (1)]. We compare the percentage of chimeras obtained with different pacemaker intensities $\psi$ and for every pacemaker intensity, we use the same set of initial conditions. It is known that the lifetime of chimeras increases with the number of oscillators $N$ (Ref. 23) and the drifting increases with decreasing $N .{ }^{25}$ Since our control aims to counteract these instabilities, we focus on small networks of up to $N=50$, and we insert a pacemaker in position $i=1$ of the network. To detect chimeras, we used the algorithm described in Appendix A. For each value of the network size, we consider all the possible values of coupling range $b$ varying from local coupling $b=1$ to global coupling $b=\frac{N-1}{2}$ when $N$ is odd, or from $b=1$ to the maximum possible value $b=\frac{N-2}{2}$ when $N$ is even. For this section, integration was performed over $4 \cdot 10^{5}$ sampling times, corresponding to $2 \cdot 10^{4}$ dimensionless time units, and all analyses were performed over an evaluation interval of 2500 dimensionless time units $I_{1}=[17500,20000]$. We considered 100 independent realizations for all pairs of values of network size $N$ and coupling range $b$. The results are displayed in Fig. 3. We clearly see that the region of the parameter space in which chimeras are detected is broader when a pacemaker is present in the network [see Figs. 3(b)-3(d)]. When no control is applied to the network [Fig. 3(a)], no chimeras are found for $N<32$ and for relative coupling range $b / N$ outside the interval $[0.25,0.4]$. This is due to the presence of chimera states whose lifetime is shorter than the integration time and to initial conditions that collapse immediately to the synchronous state without ever forming a chimera state. In the region where chimera states are present for the unchanged connectivity [Fig. 3(a)], we observe an increase in their percentage when the pacemaker is present [Figs. 3(b)-3(d)]. In particular, a low intensity pacemaker with, obtained cutting only two incoming links, already induces chimeras for small values of $N<32$ [see Fig. 3(b)]. For pacemaker intensity $\psi \approx 0.5$ [panel (c)], we obtain results that are close to the case of the full pacemaker [panel (d)].

\section{CONTROLLING THE POSITION OF CHIMERA STATES}

Secondly, we study the control of the position of chimera states. In Fig. 2(a), we see that the two complementary groups LCG and HCG drift along the network..$^{23}$ This drifting is particularly pronounced for small networks and it was characterized as a Brownian motion. ${ }^{23}$ Figures $2(\mathrm{~g})-2(\mathrm{l})$ show how the full pacemaker attracts the LCG, thus preventing its chaotic motion along the network. We study how different pacemaker intensities $\psi$ affect the chimera's position. To do this, we set $N=$ 50 and $b=18$. For these parameters, the occurrence of chimera states is more likely in comparison with smaller sizes $N$, while the drifting of the LCG and HCG is still substantial. The pacemaker is in position $i=25$. For this section, integration was performed over $2 \cdot 10^{5}$ sampling times, corresponding to $10^{4}$ dimensionless time units, and all analyses were performed over an evaluation interval of 2500 dimensionless time units $I_{2}=[7500,10000]$. Following Ref. 34, if at some point the system synchronized, we started over with new initial conditions. For every time step $t$ we define the position of the center of the LCG denoted by $l(t)$ which varies in the set $\mathcal{L}$ of numbers from 0.5 to $N=50$ in steps of 0.5 (see Appendix B). Furthermore, we calculate the size $s(t)$ of the LCG and the distance $d(t)=l(t)-25$ of its center from the pacemaker position $i=25$ (see Appendix B). In Fig. 4(a), we show the temporal (a)

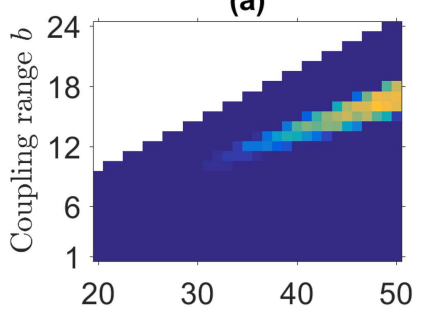

(b)

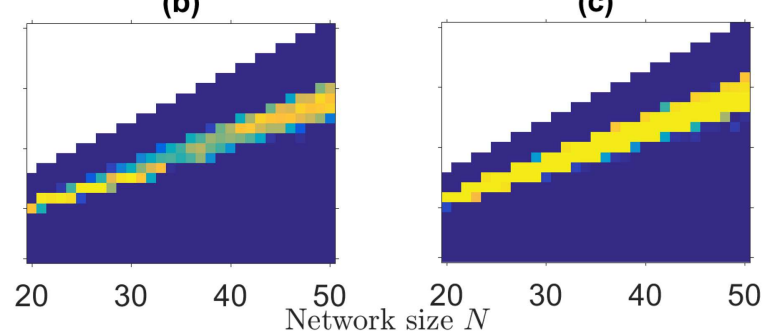

(d)

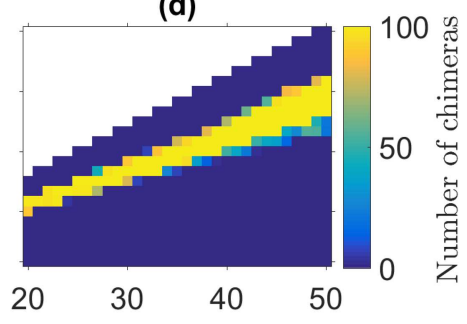

FIG. 3. A pacemaker triggers chimeras for initial conditions for which they do not form spontaneously. Comparison of the number of chimera states observed for different values of the network size $N$ and coupling broadness $b$ (the other network parameters are $\alpha=1.46, \omega=0$ ). For each pair of values, we solved the model 100 times without control [panel (a), $\psi=0$ ], with low control intensity $\psi=\frac{1}{b}$ [panel (b)], intermediate control intensity $\psi \approx 0.5$ [panel (c)] and with a full pacemaker corresponding to $\psi=1$ [panel (d)]. 
(a)

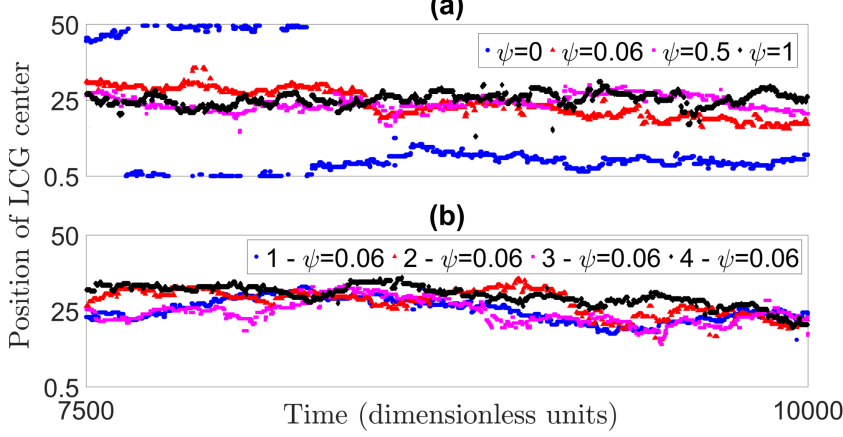

FIG. 4. The center of the LCG is attracted by pacemakers of different intensities Panel (a) is the temporal evolution of the center of the LCG for the same realization of Eq. (1) with different pacemaker intensities, starting with no control up to the full pacemaker. In panel (b), we show four independent realizations of Eq. (1) with a low pacemaker intensity.

evolution of the position of the center of the LCG for four solutions of Eq. (1) corresponding to four different pacemaker intensities $\psi=$ $0,0.06,0.5,1$, where $0.06=\frac{1}{b}$. The initial conditions were the same in every realization. In panel (b), we show four different realizations of Eq. (1) with a low pacemaker intensity $\psi=0.06$. This is the lowest possible value in our setting, as it corresponds to only two unidirectional links. In both panels, one can appreciate the attracting effect of the pacemaker on the center of the LCG. As soon as we switch on a pacemaker, even with a low intensity, the center of the LCG is attracted by the pacemaker, as it becomes evident from the difference in the characteristics of the blue to the red curve in Fig. 4(a). The control effect becomes stronger for increasing pacemaker intensity [purple and black curves in Fig. 4(a)]. In Fig. 4(b), we see how the weakest possible pacemaker with $\psi=0.06$ attracts the center of the LCG for different initial conditions, but the motion of center is more pronounced in these curves than in the black curve in panel (a), which corresponds to the full pacemaker.

Next, we study the position of the center of the LCG throughout 100 independent realizations for each pacemaker intensity. For every time step $t$ and every control intensity $\psi$, we thus have a distribution $C(l(t), \psi)$ of the position of the LCG center. For the uncontrolled system, there is no preferred position for the LCG of the chimera state over time and across different realizations. The distribution $C(l(t), 0)$, corresponding to the uncontrolled system, is shown in Fig. 5(a). As we can see from the blue curve in Fig. 5(e), the distribution $C[l(t), 0]$ is uniform on $\mathcal{L}$ during the interval $I_{2}$. As soon as we break the symmetry of the coupling topology of the oscillator network, the distribution of the position of the center changes and we see how the center position is attracted by the partial or full pacemaker. Figures 5(b)-5(d) shows the effect of increasing the pacemaker intensity $\psi$ in position 25. In Fig. 5(b), only 2 incoming links of oscillator 25 were cut, while 18 links were removed in panel (c) (corresponding to $\psi=0.06$ and $\psi=0.5$, respectively). Figure 5(d) corresponds to 36 links removed, i.e., the full pacemaker $(\psi=1)$. (a)

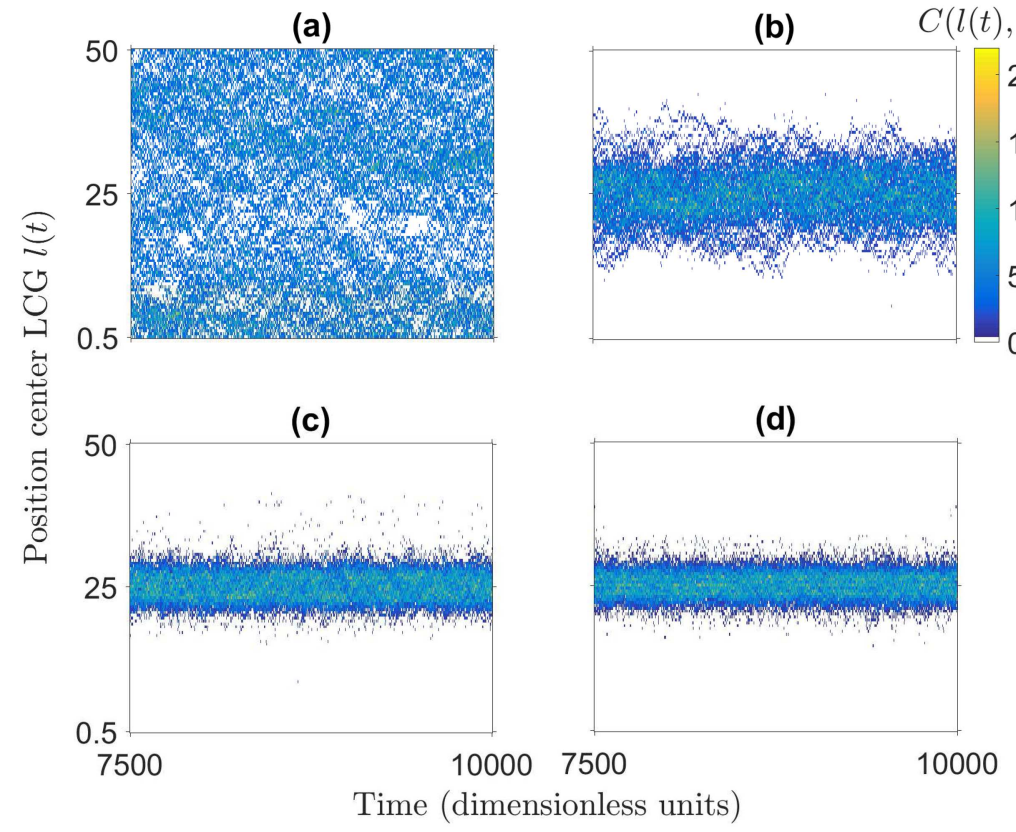

(e)

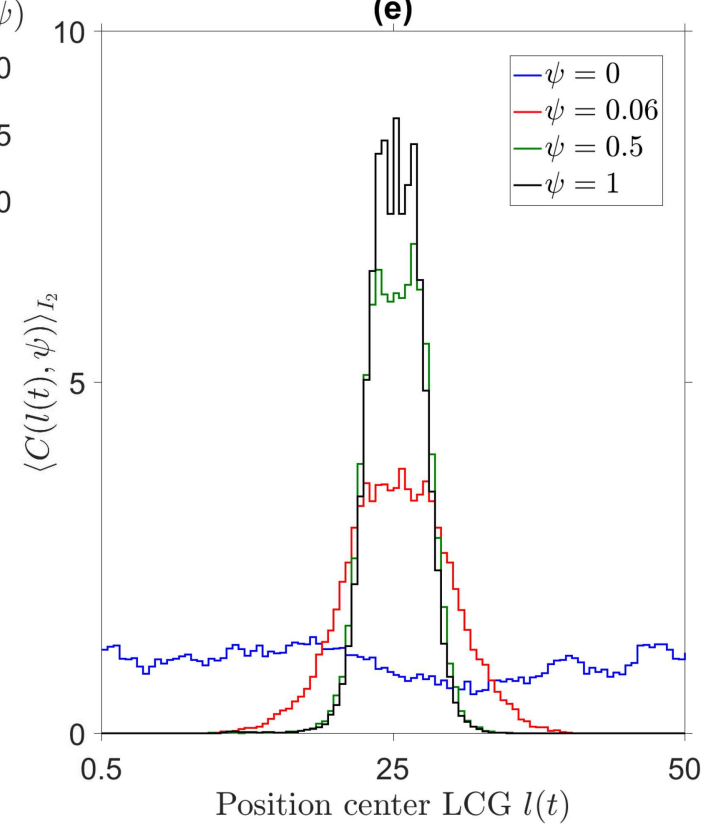

FIG. 5. Cutting incoming links of one oscillator allows one to control the position of the chimera state. We show the effects on chimera states of the presence of a partial/full pacemaker in position 25 , in a network with $N=50, b=18, \alpha=1.46$. The color scale in panels (a)-(d) represents values of the distributions of the $L C G$ center $C[I(t), \psi]$ over 100 independent realizations. Panel (a) shows how the center of the low coherence group (LCG) is positioned without any control, that is with an unmodified matrix $G$. In (b), the pacemaker intensity is $\psi=0.06$, while in (c), we have $\psi=0.5$. In panel (d), the configuration corresponding to the full pacemaker, i.e., $\psi=1$ is displayed. In panel (e), we show the corresponding time averages of the spatial distributions $C(I(t), \psi)$ of the LCG center position over the interval $I_{2}$. 
(a)

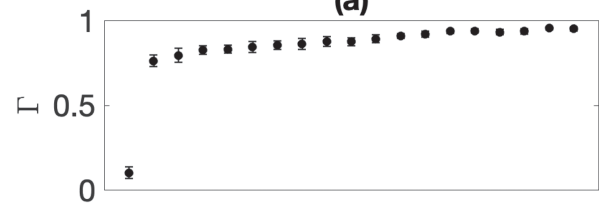

(b)

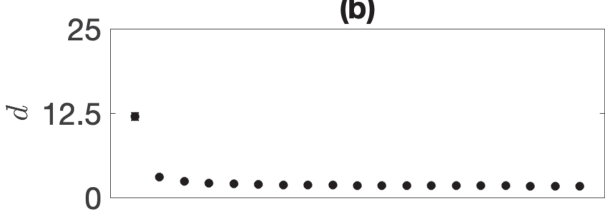

(c)

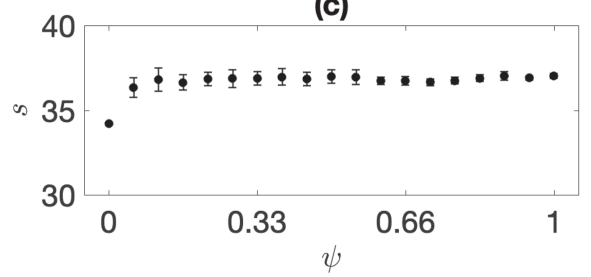

(d)

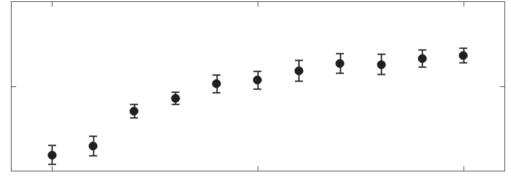

(e)

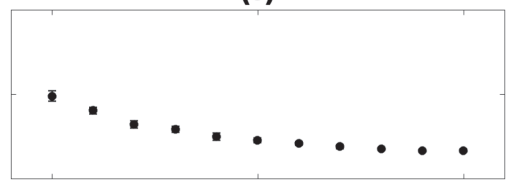

(f)

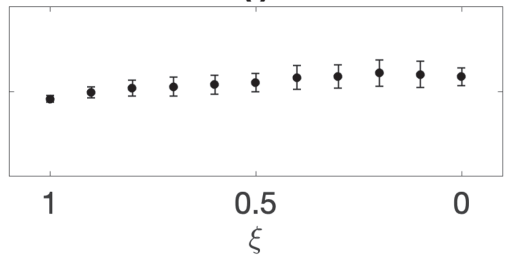

FIG. 6. Partial pacemaker is sufficient to control the chimera's position. We show effects of different control intensities on the position of chimera states. Panel (a) shows a plot of the order parameters $\Gamma(\psi)$. In panel (b), we display values of the distance $d(\psi)$ of the LCG center from the pacemaker position $i=25$. The average size of the LCG is shown in panel (c), depending on the pacemaker intensity $\psi$. Panels (d)-(f) are analogous to (a)-(c), respectively, but here $\Gamma, d, s$ are calculated for varying $\xi=G(25,7)$. For $\xi=$ 1 we have the uncontrolled system, while $\xi=0$ corresponds to one unidirectional link. The network size is $N=50$, the coupling range is $b=18$ and the phase lag is $\alpha=1.46$. All time averages were calculated over the evaluation interval $I_{2}$. The error bars display the standard deviation of the averages over the 20 sets of 100 independent initial conditions.
The control effect is clearly visible already in panel (c). Looking at the time averaged spatial distributions in panel (e), one can also observe how these become narrower with a pronounced peak around position 25 as we approach the case of the full pacemaker [see Fig. 5(e), black curve].

To further quantify the effects of our control mechanism, we define the following order parameter:

$$
\Gamma(t, \psi)=\left|\frac{1}{2 N} \sum_{l \in \mathcal{L}} C(l(t), \psi) e^{i \theta_{l}}\right|,
$$

where $\theta_{l}=\frac{2 \pi l}{N}$ for $l \in \mathcal{L}, t$ is in the evaluation interval $I_{2}$, and $|\cdot|$ is the modulus of complex numbers. We calculate the order parameter $\Gamma$ for 20 distributions of the LCG center position which were obtained from 20 sets of 100 independent initial conditions. For every pacemaker intensity $\psi$, we obtained order parameters $\Gamma_{1}(t, \psi), \ldots, \Gamma_{20}(t, \psi)$, average distances $d_{1}(t, \psi), \ldots, d_{20}(t, \psi)$, and average LCG sizes $s_{1}(t, \psi), \ldots, s_{20}(t, \psi)$. We then calculated the mean of their temporal averages over the interval $I_{2}$, thus obtaining functions of the pacemaker intensity $\Gamma(\psi), d(\psi), s(\psi)$. These values are shown in Figs. 6(a)-6(c). In panel (a), we see how the value of $\Gamma(\psi)$ sharply increases when passing from pacemaker intensity $\psi=0$ to $\psi=0.06$ (the lowest possible intensity in this setting), and then increases more slowly toward the value corresponding to the full pacemaker. The results in Fig. 6 confirm that the pacemaker attracts the LCG, in the sense that the distance $d(\psi)$ of the LCG center from the pacemaker position decreases as the pacemaker intensity $\psi$ increases. In panel (c), another effect of our control mechanism is shown: an increase in the size $s(\psi)$ of the incoherent group LCG.

The last step of our analysis is to modify only one value of $G$. We repeated the analysis described before, setting the value of the coefficient $G(25,7)=\xi$, where $\xi$ goes from 1 (unchanged matrix $G$ ) to 0 (one unidirectional link). The results are represented in Figs. 6(d)-6(f). In panel (d), we observe an increase of the order parameter $\Gamma(\xi)$ when the modification of the coupling matrix becomes stronger. In particular we see that, as we decrease the value of $G(25,7)=\xi$ (which corresponds to increasing the control intensity), the distribution of the position of the LCG center becomes more and more similar to the one obtained in Fig. 5(b), where two links were made unidirectional, as it is reflected in the increasing values of $\Gamma(\xi)$ [Fig. 6(d)] and the decreasing values of the distance $d(\xi)$ of the LCG center from the pacemaker [Fig. 6(e)]. The effect on the size of the LCG shown in Fig. 6(f) is not as pronounced as it was in the case of the transition from no control to the full pacemaker.

\section{ALTERNATIVE SYMMETRY BREAKING MECHANISM}

To further understand which are the important aspects of the control mechanism introduced in this paper, we analyze here what happens when we reverse the pacemaker idea. That is, we select one oscillator with index $i$ and we cut all of its outgoing links. In terms of the coupling matrix $G$ of Eq. (2), this corresponds to setting $G(j, i)=0$ for a fixed column $i$ and for all $j \neq i$. This new configuration is equivalent to isolating oscillator $i$ from the rest of the network, but we continue to show its dynamics in our results. Figure 7 shows what happens when we repeat the simulations of Fig. 2 substituting the pacemaker with the new symmetry breaking configuration which consists in cutting the outgoing link of oscillator 18. Panels (a)-(c) of Fig. 7 are replicas of (a)-(c) of Fig. 2 (uncontrolled chimeras), and we can see how the remaining panels of Fig. 7 are qualitatively similar to the corresponding panels obtained in Fig. 2 using the full pacemaker. These findings provide further evidence that the essential element for chimera control is the disruption of the spatial symmetry of the ring network. 
(a)

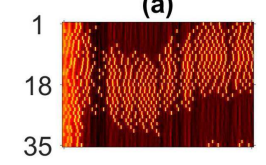

(b)

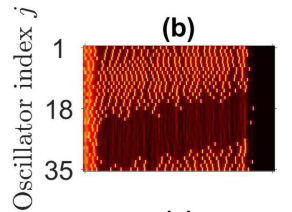

(c)

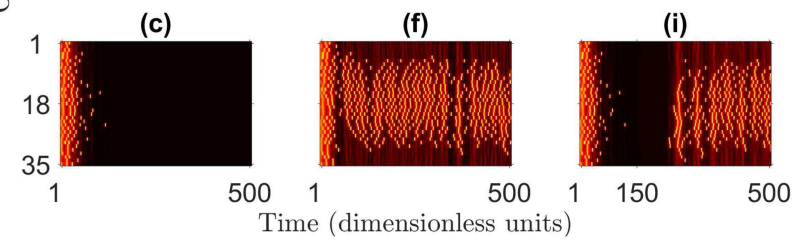

FIG. 7. Cutting the outgoing links of one oscillator acts like a full pacemaker. In panels (a)-(c), we display instantaneous phase velocities for three different realizations of Eq. (1) for $N=35, b=12, \alpha=1.46, \omega=0$ (same initial conditions as in Fig. 2). In panels (g)-(i), we display the effects of the symmetry breaking described in Sec. VI with $i=18$ on the solutions shown in panels (a)-(c), respectively. The symmetry breaking was activated at the beginning of the simulations. Panels (j)-(I) are analogous to panels ( $g$ )-(i), but here the symmetry breaking was activated after 150 dimensionless time units.

\section{DISCUSSION}

We introduced a method based on the idea of a pacemaker oscillator which allows one to control chimera states in small networks of phase oscillators. By varying the control intensity, we were able to investigate which is the minimal action needed to control chimera states. We found that modifying only one coefficient in the connectivity matrix is enough to control the chimera's position. Appealing features of our method are the simplicity of its implementation, which lies in the fact that no feedback from the system is needed and that it does not intervene on the oscillators' parameters. Interestingly, there are strong analogies between our results and the ones elaborated by Isele et al. ${ }^{29}$ Although they use a different model and a completely different control mechanism, they also observed that the symmetry breaking element in the network attracts the incoherent group and stabilizes the chimera state. The effects of symmetry breaking in the evolution of chimera states also emerge in the recent work by Yao et al. in Ref. 35. They perturbed the dynamics of a ring-shaped network of phase oscillators by selecting a target oscillator and forcing it to have a fixed phase difference with respect to the local mean field of its neighbors. This perturbation induces the incoherent group to be centered around the target oscillator. Our results confirm the occurrence of this self-adaptation ${ }^{35}$ of the chimera position and generalize the findings in Ref. 35 showing that weaker changes in the network are sufficient not only to control the chimera's position, but also to trigger chimeras for parameters and initial conditions for which they do not form spontaneously. Moreover, the full pacemaker can be used to generate a chimera state after the system has collapsed to synchronous solution, as we show in Fig. 2.
It is worth to point out that the idea of a pacemaker was already introduced in the study of synchronization of the Kuramoto model. In Refs. 36 and 37, a pacemaker is used to synchronize random networks of phase oscillators. We showed that the same mechanism produces the opposite effect for the Kuramoto-Sakaguchi model. In fact, it promotes the existence of chimera states when the oscillators are nonlocally coupled. This comparison underlines the importance of the interplay of nonlocal coupling, the phase lag, and the control mechanism in the control of chimera states. Given that our method acts exclusively on the connectivity of the network and not on the intrinsic dynamics of the oscillators, we conjecture that it may work also for networks made of different types of oscillators and more complex topologies.

\section{ACKNOWLEDGMENTS}

This project was supported by the Volkswagen Foundation, the Spanish Ministry of Economy and Competitiveness (Grant No. FIS2014-54177-R), the CERCA Program of the Generalitat de Catalunya (G.R. and R.G.A.), and the European Union's Horizon 2020 program under the Marie Sklodowska-Curie Grant Agreement No. 642563 (R.G.A). We acknowledge support from the Deutsche Forschungsgemeinschaft (DFG) in the framework of the SFB 910, Projektnummer 163436311 (I.O., E.S., and A.Z.).

\section{APPENDIX A: ALGORITHM FOR CHIMERA DETECTION}

We used the following algorithm for the detection of chimera states. ${ }^{29}$ We integrate Eq. (1) to obtain a solution $\phi_{j}(t)$ for 400000 time steps of width $d t=0.05$. In the absence of control, we calculate the Kuramoto global order parameter

$$
R(t)=\frac{1}{N} \sum_{k=1}^{N} e^{i \phi_{k}(t)},
$$

and we compute its temporal average $\left.R=\langle R(t)\rangle_{I_{1}}\right]$ over the evaluation interval $I_{1}=[17500,20000]$ described in Sec. IV. We compute the mean phase velocities

$$
\Omega_{j}=\left\langle\frac{\mathrm{d} \phi_{j}(t)}{d t}\right\rangle_{I_{1}} \text { for } j=1, \ldots, N,
$$

and we determine the range of the mean phase velocity profile:

$$
\Omega=\max _{j=1, \ldots, N} \Omega_{j}-\min _{j=1, \ldots, N} \Omega_{j} .
$$

In the case an oscillator acts as a pacemaker, we exclude this oscillator from the averaging.

If we find that $R \in[0.65,0.8]$ and $\Omega \in[0.1,1]$, then the solution $\phi_{j}(t)$ is classified as a chimera state. These threshold values are based on preanalysis results. The other possible scenarios for Eq. (1) are solutions which are completely incoherent and solutions in which the oscillators are all synchronized or almost all synchronized. The former are discarded by the lower bound on the order parameter. The latter are also ruled out because $R=1$ if all oscillators are synchronized. The condition on the mean phase velocity comes into play when we have values of $R$ close to 0.65 . In this case, in the solution there is no clear distinction between coherent and incoherent group and $\Omega<0.1$. The upper-bound for $\Omega$ discards situations that 
are rarely observed in the presence of high coupling and a pacemaker, in which a chimera state is not formed but the synchronized state is disturbed by few oscillators that have a different frequency from the synchronized block.

\section{APPENDIX B: HCG AND LCG GROUPS}

In the analysis presented in Sec. V, we used the concepts of high coherence group (HCG) and low coherence group (LCG) that form a chimera state. These two groups were defined following the algorithm presented in Ref. 19. In what follows, all indexes and sums of indexes are to be understood modulo $N$. For the $j$-th oscillator, we consider its two nearest neighbors on each side, that is oscillators $j-2, j-1, j+1, j+2$. For every time instant $t$, we calculate the pairwise local order parameters $R_{j+2, j+1}(t), R_{j+1, j}(t), R_{j, j-1}(t)$, $R_{j-1, j-2}(t)$, where

$$
R_{a, b}(t)=\left|\frac{1}{2}\left(e^{i \phi_{a}(t)}+e^{i \phi_{b}(t)}\right)\right| .
$$

We define the following function:

$$
\chi(j, t)= \begin{cases}1 \quad \text { if } R_{j+2, j+1}(t), R_{j+1, j}(t), \\ R_{j, j-1}(t) \text { and } R_{j-1, j-2}(t)>0.995 \\ 0 \quad \text { otherwise. }\end{cases}
$$

At time $t$, the HCG is formed by all oscillators with indexes $j$ such that $\chi(j, t)=1$, the LCG is formed by the remaining oscillators. Once we defined the HCG and LCG, we can define the border of the LCG and its center. For every time $t \in I_{2}$, we look for indexes $i_{b}, j_{b}$ which satisfy the following conditions:

$$
\begin{aligned}
& \chi\left(i_{b}-1, t\right)=1 \text { and } \chi\left(i_{b}, t\right)=\chi\left(i_{b}+1, t\right)=0, \\
& \chi\left(j_{b}-1, t\right)=\chi\left(j_{b}, t\right)=0 \text { and } \chi\left(j_{b}+1, t\right)=1 .
\end{aligned}
$$

If such indices exist, we say that the border of the LCG is $B(t)=\left\{i_{b}, j_{b}\right\}$. Apart from the main $\mathrm{LCH}$, it may happen that there are small islands of incoherent oscillators inside the HCG. In this case, we find multiple pairs of indexes $i_{b}^{1}, j_{b}^{1}, i_{b}^{2}, j_{b}^{2}, \ldots, i_{b}^{n}, j_{b}^{n}$ satisfying the conditions above. We choose index $k$ corresponding to the biggest incoherent group and the border is $B(t)=\left\{i_{b}^{k}, j_{b}^{k}\right\}$. The position $l(t)$ of the center of the LCG at time $t$ is defined according to the following rule:

- if $i_{b}<j_{b}$, then $l(t)=\frac{i_{b}+j_{b}}{2}$,

- if $i_{b}>j_{b}$, then $l(t)=\frac{i_{b}+j_{b}+N}{2} \bmod 50$.

The center position $l(t)$ defined above can be an integer or halfinteger between 0.5 and the network size $N$. The size of the LCG at time $t$ is $s(t)=50-\sum_{k=1}^{50} \chi(i, t)$.

\section{REFERENCES}

${ }^{1}$ Y. Kuramoto and D. Battogtokh, "Coexistence of coherence and incoherence in nonlocally coupled phase oscillators," Nonlinear Phenom. Complex Syst. 4, 380-385 (2002).

${ }^{2}$ D. M. Abrams and S. H. Strogatz, "Chimera states for coupled oscillators," Phys. Rev. Lett. 93, 174102 (2004).

${ }^{3}$ J. Hizanidis, V. G. Kanas, A. Bezerianos, and T. Bountis, "Chimera states in networks of nonlocally coupled Hindmarsh-Rose neuron models," Int. J. Bifurcat. Chaos 24, 1450030 (2014).
${ }^{4}$ I. Omelchenko, O. E. Omel'chenko, P. Hövel, and E. Schöll, "When nonlocal coupling between oscillators becomes stronger: Patched synchrony or multichimera states," Phys. Rev. Lett. 110, 224101 (2013).

${ }^{5}$ L. Schmidt, K. Schönleber, K. Krischer, and V. García-Morales, "Coexistence of synchrony and incoherence in oscillatory media under nonlinear global coupling," Chaos 24, 013102 (2014).

${ }^{6}$ A. Zakharova, M. Kapeller, and E. Schöll, "Amplitude chimeras and chimera death in dynamical networks,” J. Phys. Conf. Ser. 727, 010218 (2016).

${ }^{7}$ A. Gjurchinovski, E. Schöll, and A. Zakharova, "Control of amplitude chimeras by time delay in oscillator networks," Phys. Rev. E 95, 042218 (2017).

${ }^{8}$ A. zur Bonsen, I. Omelchenko, A. Zakharova, and E. Schöll, "Chimera states in networks of logistic maps with hierarchical connectivities," Eur. Phys. J. B 91, 65 (2018).

${ }^{9}$ T. Chouzouris, I. Omelchenko, A. Zakharova, J. Hlinka, P. Jiruska, and E. Schöll, "Chimera states in brain networks: Empirical neural vs. modular fractal connectivity," Chaos 28, 045112 (2018).

${ }^{10}$ A. M. Hagerstrom, T. E. Murphy, R. Roy, P. Hoevel, I. Omelchenko, and E. Schoell, "Experimental observation of chimeras in coupled-map lattices," Nat. Phys. 8, 658-661 (2012).

${ }^{11}$ M. R. Tinsley, S. Nkomo, and K. Showalter, "Chimera and phase-cluster states in populations of coupled chemical oscillators,” Nat. Phys. 8, 662-665 (2012).

${ }^{12}$ L. Larger, B. Penkovsky, and Y. Maistrenko, "Virtual chimera states for delayedfeedback systems," Phys. Rev. Lett. 111, 054103 (2013).

${ }^{13}$ E. A. Martens, S. Thutupalli, A. Fourrière, and O. Hallatschek, "Chimera states in mechanical oscillator networks," Proc. Natl. Acad. Sci. U.S.A. 110, 10563-10567 (2013).

${ }^{14} \mathrm{M}$. Wickramasinghe and I. Z. Kiss, "Spatially organized dynamical states in chemical oscillator networks: Synchronization, dynamical differentiation, and chimera patterns," PLoS One 8, e80586 (2013).

${ }^{15}$ L. V. Gambuzza, A. Buscarino, S. Chessari, L. Fortuna, R. Meucci, and M. Frasca, "Experimental investigation of chimera states with quiescent and synchronous domains in coupled electronic oscillators," Phys. Rev. E 90, 032905 (2014).

${ }^{16} \mathrm{~J}$. F. Totz, J. Rode, M. R. Tinsley, K. Showalter, and H. Engel, "Spiral wave chimera states in large populations of coupled chemical oscillators," Nat. Phys. 14, 282-285 (2018).

${ }^{17}$ J. C. González-Avella, M. G. Cosenza, and M. San Miguel, "Localized coherence in two interacting populations of social agents," Phys. A Stat. Mech. Appl. 399, 24-30 (2014).

${ }^{18}$ V. M. Bastidas, I. Omelchenko, A. Zakharova, E. Schöll, and T. Brandes, "Quantum signatures of chimera states," Phys. Rev. E 92, 062924 (2015).

${ }^{19}$ R. G. Andrzejak, C. Rummel, F. Mormann, and K. Schindler, "All together now: Analogies between chimera state collapses and epileptic seizures," Sci. Rep. 6, 23000 (2016).

${ }^{20}$ T. Banerjee, P. S. Dutta, A. Zakharova, and E. Schöll, "Chimera patterns induced by distance-dependent power-law coupling in ecological networks," Phys. Rev. E 94, 032206 (2016).

${ }^{21}$ N. E. Kouvaris, R. J. Requejo, J. Hizanidis, and A. Díaz-Guilera, "Chimera states in a network-organized public goods game with destructive agents," Chaos 26, 123108 (2016).

${ }^{22}$ O. E. Omel'chenko, "The mathematics behind chimera states," Nonlinearity $\mathbf{3 1}$, R121 (2018).

${ }^{23} \mathrm{M}$. Wolfrum and O. E. Omel'chenko, "Chimera states are chaotic transients," Phys. Rev. E 84, 015201 (2011).

${ }^{24} \mathrm{~J}$. Sieber, O. E. Omel'chenko, and M. Wolfrum, "Controlling unstable chaos: Stabilizing chimera states by feedback,” Phys. Rev. Lett. 112, 054102 (2014).

${ }^{25}$ O. E. Omel'chenko, M. Wolfrum, and Y. L. Maistrenko, "Chimera states as chaotic spatiotemporal patterns," Phys. Rev. E 81, 065201 (2010).

${ }^{26}$ C. Bick and E. A. Martens, "Controlling chimeras," New J. Phys. 17, 33030 (2015).

${ }^{27}$ I. Omelchenko, O. E. Omel'chenko, A. Zakharova, M. Wolfrum, and E. Schöll, "Tweezers for chimeras in small networks," Phys. Rev. Lett. 116, 114101 (2016).

${ }^{28}$ L. V. Gambuzza and M. Frasca, "Pinning control of chimera states," Phys. Rev. E 94, 022306 (2016)

${ }^{29}$ T. Isele, J. Hizanidis, A. Provata, and P. Hövel, "Controlling chimera states: The influence of excitable units,” Phys. Rev. E 93, 022217 (2016). 
${ }^{30}$ P. Kalle, J. Sawicki, A. Zakharova, and E. Schöll, "Chimera states and the interplay between initial conditions and non-local coupling," Chaos 27, 033110 (2017).

${ }^{31}$ I. Omelchenko, O. E. Omel'chenko, A. Zakharova, and E. Schöll, "Optimal design of tweezer control for chimera states," Phys. Rev. E 97, 012216 (2018).

${ }^{32}$ Y. Suda and K. Okuda, "Persistent chimera states in nonlocally coupled phase oscillators,” Phys. Rev. E 92, 060901 (2015).

${ }^{33}$ C. Bick, M. Sebek, and I. Z. Kiss, "Robust weak chimeras in oscillator networks with delayed linear and quadratic interactions," Phys. Rev. Lett. 119, 168301 (2017).
${ }^{34}$ R. G. Andrzejak, G. Ruzzene, and I. Malvestio, "Generalized synchronization between chimera states," Chaos 27, 053114 (2017).

${ }^{35}$ N. Yao, Z.-G. Huang, H.-P. Ren, C. Grebogi, and Y.-C. Lai, "Self-adaptation of chimera states,” Phys. Rev. E 99, 010201 (2019).

${ }^{36} \mathrm{H}$. Kori and A. S. Mikhailov, "Entrainment of randomly coupled oscillator networks by a pacemaker," Phys. Rev. Lett. 93, 254101 (2004).

${ }^{37}$ F. Radicchi and H. Meyer-Ortmanns, "Entrainment of coupled oscillators on regular networks by pacemakers," Phys. Rev. E 73, 036218 (2006).

${ }^{38}$ R. G. Andrzejak, G. Ruzzene, I. Malvestio, K. Schindler, E. Schöll, and A. Zakharova, "Mean field phase synchronization between chimera states," Chaos 28, 091101 (2018). 